\title{
суицидология
}

УДК 616.89-008.1:616.89-008.441.44(470.3)(=1.2)(06)

Для цитирования: Носова Е.С., Жуков И.В., Радулов С.П. Психические расстройства и суицидальное поведение: результаты анализа медицинской документации. Сибирский вестник психиатрии и наркологии. 2021; 2 (111): 54-63. https://doi.org/10.26617/1810-3111-2021-2(111)-54-63

\section{Психические расстройства и суицидальное поведение: результаты анализа медицинской документации}

\section{Носова Е.С., Жуков И.В., Радулов С.П.}

ГБУЗ КО «Калужская областная психиатрическая больница им. А.Е. Лифиица»

Россия, 248009, Калуга, ул. Маяковского, 55

\section{PEЗЮME}

Психические расстройства - один из наиболее изученных факторов риска суицидального поведения. Степень выраженности суицидального риска при психических заболеваниях варьирует в зависимости от диагноза и демографических характеристик [1], что обозначает направление для дальнейших исследований вопроса на локальном уровне. Цель: изучение нозологической структуры поступлений в психиатрический стационар в связи с суицидальным поведением и идентификация уязвимых групп для разработки профилактических мероприятий. Материалы и методы: на основе анализа архивной медицинской документации ГБУЗ КО «Калужская областная психиатрическая больница им. А.Е. Лифшица» за 2017-2018 гг. изучено 435 случаев госпитализаций пациентов с суицидальным поведением. При формировании выборки использована методика К.А. Отдельновой (1980) для исследования повышенной точности (t=2,0; p=0,95) [2]. Статистическая обработка данных проводилась с использованием статистических таблиц и приложения Microsoft Excel. Результаты: по частоте встречаемости диагностические рубрики расположились следующим образом: F20-F29 (37,47\%), F00-F09 (17,7\%), F10-F19 (12,64\%), F90-F98 (8,97\%), F70-F79 (8,5\%), F30-F39 (7,13\%), менее 10\% иные рубрики. Доказательно показано, что рубрика F70-F79 и рубрика F21 статистически значимо сопряжены с риском госпитализации с суицидальными мыслями, шифр F10.2 - с риском суицидальной попытки. Первичные поступления чаще наблюдаются в группе пациентов с суицидальными попытками, повторные в группе пациентов с суицидальными мыслями. Однако с позиций диагноза статистически значимой является зависимость только в рубриках F30-F39 и F10-F19, первичные поступления пациентов с этими психиатрическими диагнозами чаще были обусловлены суицидальными попытками. Выводы. Обсуждаемый фактический материал исследования дает общее представление о наиболее распространенных психических расстройствах, сопровождающих суицидальное поведение госпитализированных пациентов. Представленная нозологическая структура госпитализаций определяет необходимость развития реабилитационных мероприятий, оптимизации терапевтических интервенций и усиления социальной поддержки пациентов. Оптимальны дальнейшие исследования клинико-социальных характеристик суицидентов, а также механизмов суицидального поведения при разных психических расстройствах для улучшения понимания модели суицидального поведения популяции региона на примере населения Калуги, входящей в Центральный федеральный округ.

Ключевые слова: психические и поведенческие расстройства, суицид, суицидальные мысли, суицидальная попытка, суицидальное поведение, эпидемиология, факторы риска.

\section{ВВЕДЕНИЕ}

Ежегодные потери от самоубийств составляют около 800000 смертей, при этом 79\% всех суицидов приходится на страны с низким и средним уровнями дохода населения [3]. Суициды обусловливают около 1,4\% мировой смертности с колебанием показателя от $0,5 \%$ в государствах африканского континента до $1,9 \%$ в ЮгоВосточной Азии (здесь и далее - регионы по классификации ВО3) [4]. В 2016 г. потери от суицидальной смертности составили 34,6 млн лет утраченной жизни на 100000 населения и обо- значили первенство бремени суицидов в странах Азиатско-Тихоокеанского региона с высоким уровнем доходов, занимая также лидирующие позиции среди иных причин в Восточной Европе и Океании, Центральной и Западной Европе, а также Северной Америке [5].

Известно, что психические расстройства являются фактором риска суицидального поведения. Так, в соответствии с данными психологических аутопсий более чем в $80 \%$ случаев суицидентам был установлен психиатрический диагноз [6]. 
Предположительным механизмом такой ассоциации авторы считают возможный общий этиопатогенез психических расстройств и суицидального поведения в рамках биопсихосоциальной парадигмы [7]. Дефицит навыков совладания с кризисными ситуациями и несовершенство копинг-стратегий [8], маргинализация лиц с психическими расстройствами, их стигматизация, а также отсутствие социальной поддержки, социальная дезинтеграция, одиночество [9], безработица [10] способствуют росту суицидального риска. Как показано в ряде исследований, сочетание нескольких психиатрических диагнозов увеличивает суицидальный риск $[11,12]$, а пережитый психотический опыт повышает риск суицидальной попытки как при установленном психиатрическом диагнозе, так и без него [13].

Испытанием для системы здравоохранения многих стран мира в 2020 г. стала пандемия COVID-19, на сегодняшний день унесшая жизни более 2,6 млн человек [14]. Социальная изоляция, тревога, страх заражения, хронический стресс и финансовые трудности относятся к числу потенциальных триггеров развития депрессивных, тревожно-депрессивных расстройств, психических и поведенческих расстройств, связанных с употреблением психоактивных веществ в кластере уязвимых лиц, а также экзацербации уже имеющегося процесса [15]. Обсуждается предположительный рост числа самоубийств в ближайшей перспективе и предлагаются стратегии профилактики, возможные для осуществления в вынужденных ограничительных условиях. Отмечена настоятельная потребность в повторных кросссекционных и продольных исследованиях групп риска для разработки прицельных превентивных мероприятий [16].

\section{ЦЕЛЬ ИССЛЕДОВАНИЯ}

Изучение нозологической структуры поступлений пациентов в психиатрический стационар в связи с суицидальным поведением и идентификация уязвимых групп для разработки профилактических интервенций.

\section{МАТЕРИАЛЫ И МЕТОДЫ}

На основе анализа архивной медицинской документации ГБУЗ КО «Калужская областная психиатрическая больница им. А.Е. Лифшица» за 2017-2018 гг. выявлено 435 случаев госпитализации пациентов с суицидальным поведением. Данные выкопированы из «Медицинских карт стационарного больного» (форма № 003/y). При формировании выборки была использована методика К.А. Отдельновой (1980) для исследования повышенной точности $(\mathrm{t}=2, \mathrm{p}=0,95)$.

Статистическая обработка данных проводилась с использованием статистических таблиц и приложения Microsoft Excel.

\section{РЕЗУЛЬТАТЫ}

Общее число госпитализаций за двухлетний период (2017-2018 гг.) составило 9859 случаев, из них 435 были обусловлены суицидальным поведением пациентов. Все поступления были распределены на два кластера: госпитализации, связанные с суицидальными мыслями (далее - CM), и поступления в связи с суицидальными попытками и самоповреждениями, с уточненными суицидальными намерениями (далее - СП). Соотношение пациентов с СП и СМ оказалось идентичным - 1:1, составляя 218 и 217 случаев соответственно

При поступлении из общего массива случаев так называемыми первичными поступлениями для врача-психиатра были 175 пациентов, что составляет менее половины от всех госпитализаций $(40,2 \%)$. В то же время большинство пациентов ранее уже пользовались психиатрической помощью, из них основная часть на момент поступления находилась под диспансерным наблюдением у врача-психиатра (47\%), 40\% получало психиатрическую помощь по обращаемости, 4\% наблюдалось в группе активного диспансерного наблюдения, на учёте у нарколога состояло $8 \%, 1 \%$ оказывалась помощь врача-психиатра в психоневрологическом интернате по месту жительства.

Следует отметить, что первичным пациентам при выписке из стационара более чем в половине случаев была рекомендована помощь врачапсихиатра по обращаемости (60\%), удельный вес перенаправленных к наркологу - 17,1\%, диспансерный учёт рекомендован практически четверти первичных больных (22,9\%).

Согласно фактическому материалу нозологическая структура выборки выглядела следующим образом (рис. 1).

Так, среди всех диагностических рубрик наибольшее количество поступлений приходилось на пациентов с шизофренией, шизотипическими и бредовыми расстройствами $(37,47 \%)$. Второе место занимали пациенты с органическими расстройствами, включая симптоматические психические расстройства $(17,7 \%)$. 8,5\% заключительных диагнозов относились к рубрике F70F79, 12,64\% - к рубрике F10-F19. 7,13\% поступлений составили пациенты с аффективными расстройствами. Эмоциональные расстройства и расстройства поведения, начинающиеся в детском и подростковом возрасте, зарегистрированы у $8,97 \%$ пациентов $(\mathrm{n}=39)$. Менее $10 \%$ случаев поступления пациентов в психиатрический стационар распределено в остальных рубриках МКБ-10.

Более подробно, с различиями по виду суицидального поведения, рубрики рассмотрены в таблице 1. 


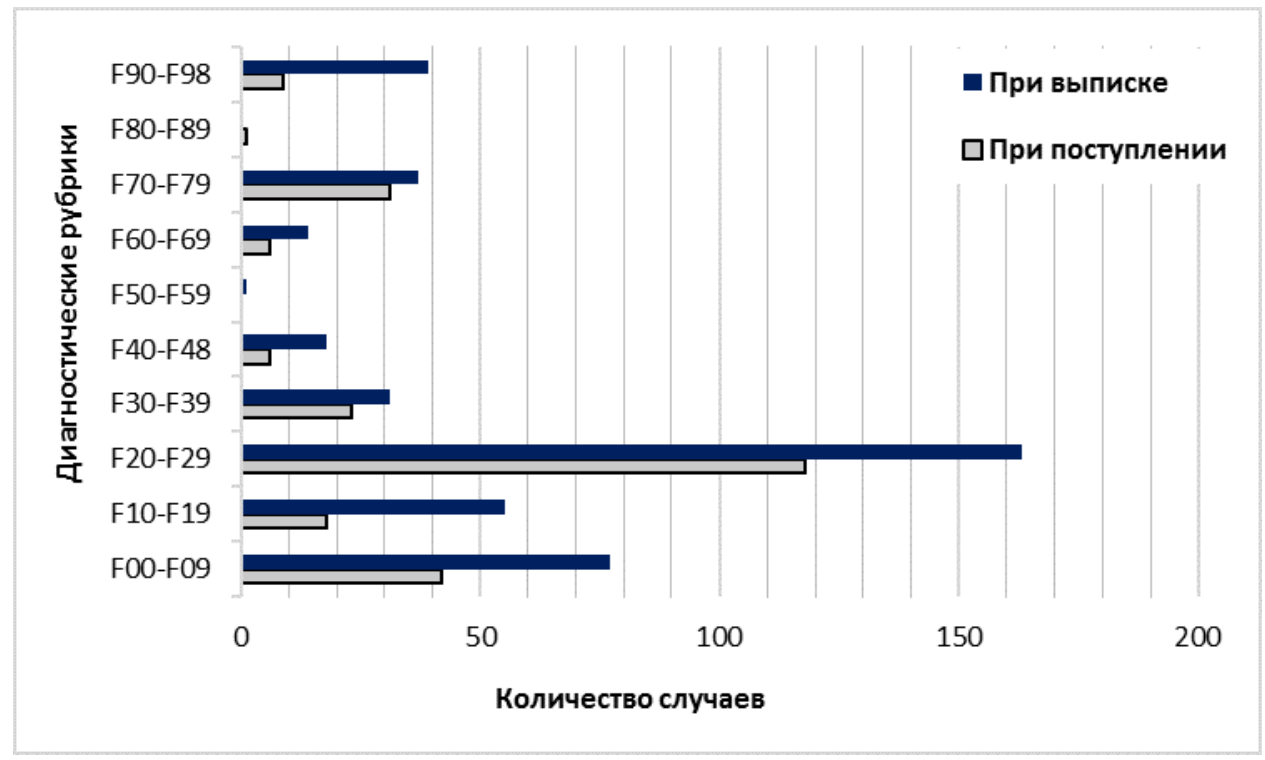

Р и с у н о к 1. Нозологическая структура поступлений пациентов с суицидальным поведением в ГБУЗ КО «КОПБ им. А.Е. Лифшица» в 2017-2018 гг., кол-во случаев

В кластере «при поступлении» не фигурируют первичные пациенты

Т а б л и ц а 1. Распределение поступлений пациентов с суицидальным поведением в ГБУЗ КО «КОПБ им. А.Е. Лифшица» в 2017-2018 гг. в зависимости от диагностической рубрики МКБ-10, кол-во случаев

\begin{tabular}{|c|c|c|c|}
\hline Код по МКБ-10 & СМ & СП & Итого \\
\hline F00-F09 & 36 & 41 & 77 \\
\hline F10-F19 & 16 & 39 & 55 \\
\hline F20-F29 & 90 & 73 & 163 \\
\hline F30-F39 & 15 & 16 & 11 \\
\hline F40-F48 & 7 & 0 & 18 \\
\hline F50-F59 & 1 & 10 & 1 \\
\hline F60-F69 & 4 & 8 & 37 \\
\hline F70-F79 & 29 & 0 & 0 \\
\hline F80-F89 & 0 & 20 & 39 \\
\hline F90-F98 & 19 & 218 & 435 \\
\hline Всего & 217 & & \\
\hline
\end{tabular}

Согласно результатам анализа фактического материала в кластере СМ преобладали поступления пациентов с диагнозами из рубрики F70-F79, а в группе пациентов с СП - из рубрик F00-F09 и F10-F19. Далее была выполнена проверка гипо- тезы Н0: ру=рх при конкурирующей гипотезе Н1: py>px $(\mathrm{y}-\mathrm{CM}, \mathrm{x}-\mathrm{C \Pi})$.

Наблюдаемое значение критерия продемонстрировано в таблице 2 .

Т а б л и ц а 2. Расчетные данные, использованные для определения частоты встречаемости диагностических рубрик МКБ-10 у пациентов с суицидальным поведением

\begin{tabular}{|c|c|c|c|c|c|c|}
\hline \multirow{2}{*}{$\begin{array}{c}\text { Код } \\
\text { по МКБ-10 } \\
\end{array}$} & \multirow{2}{*}{$\mathrm{CM}$} & \multirow{2}{*}{$\mathrm{C} \Pi$} & \multirow{2}{*}{$\mathrm{p}$} & \multicolumn{2}{|c|}{ Относительные частоты } & \multirow{2}{*}{$\begin{array}{c}\mathrm{U} \\
\text { наблюдений }\end{array}$} \\
\hline & & & & $\mathrm{CM}$ & $\mathrm{C \Pi}$ & \\
\hline F00-F09 & 36 & 41 & 0,1770 & 0,1659 & 0,1881 & $-0,6066$ \\
\hline F10-F19 & 16 & 39 & 0,1263 & 0,0737 & 0,1789 & $-3,3025$ \\
\hline F20-F29 & 90 & 74 & 0,3748 & 0,4147 & 0,3349 & 1,7191 \\
\hline F30-F39 & 15 & 16 & 0,0713 & 0,0691 & 0,0734 & $-0,1743$ \\
\hline F40-F48 & 7 & 11 & 0,0414 & 0,0323 & 0,0505 & $-0,9527$ \\
\hline F50-F59 & 1 & 0 & 0,0023 & 0,0046 & 0,0000 & 1,0014 \\
\hline F60-F69 & 4 & 10 & 0,0322 & 0,0184 & 0,0459 & $-1,6245$ \\
\hline F70-F79 & 29 & 8 & 0,0852 & 0,1336 & 0,0367 & 3,6195 \\
\hline F80-F89 & 0 & 1 & 0,0000 & 0,0000 & 0,0000 & - \\
\hline F90-F98 & 19 & 20 & 0,0897 & 0,0876 & 0,0917 & $-0,1496$ \\
\hline
\end{tabular}


Т.е. при конкурирующей гипотезе $\mathrm{H} 1 \mathrm{p} 1>\mathrm{p} 2$, $\mathrm{u}_{\mathrm{kp}}$ для правосторонней критической области находится из условия:

$$
\Phi\left(u_{\kappa p}\right)=\frac{1-2 \alpha}{2}
$$

Соответственно вид критической области:

$$
\mathrm{U}>\mathrm{u}_{\mathrm{\kappa p}}, \quad \Phi\left(u_{\kappa p}\right)=\frac{1-2 \cdot 0,05}{2}=0,45,
$$

Следовательно, $\mathrm{u}_{\mathrm{\kappa p}}=1,645$.

Далее были рассмотрены зарегистрированные

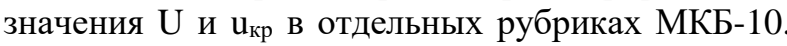
Для рубрики F10-F19 U=-3,3025<u кр $=-1,645$. Следовательно, гипотеза Н0 не принимается, в связи с чем можно считать, что во всей генеральной совокупности вероятность поступления пациентов с СМ в категории F10-F19 меныше вероятности поступления пациентов с СП.

Для рубрики F70-F79 U=3,6195> $\mathrm{u}_{\mathrm{\kappa p}}=1,645$. Coответственно гипотеза Н0 так же не принимается, поэтому можно считать, что во всей генеральной совокупности вероятность госпитализации паци- ентов с CM в категории F70-F79 больше вероятности госпитализации пациентов с СП.

B категории F20-F29 гипотеза H0, как и в двух предыдущих случаях, по данному критерию не принимается, однако доверительные интервалы (по критерию Стьюдента, а также при меньшем уровне значимости) пересекаются. Таким образом, нельзя с определенной точностью сказать, что поступление пациентов с СМ с диагнозом шизофрении, шизотипических и бредовых расстройств превалирует над поступлением пациентов с СП. Вместе с тем в категории F20-F29, включающей подкатегорию F21, госпитализация пациентов с СМ встречается в генеральной совокупности с более высокой частотой, чем пациентов с СП.

В таблице 3 приведены подкатегории и конкретные диагнозы, вызывающие интерес при изучении частоты встречаемости СМ и СП при стационарной госпитализаций пациентов с суицидальным поведением.

Т а б л и ц а 3. Расчетные данные для определения зависимости вида суицидального поведения от диагноза у пациентов ГБУЗ КО «КОПБ им. А.Е. Лифшица» в 2017-2018 гг.

\begin{tabular}{|c|c|c|c|c|c|c|}
\hline Код по МКБ-10 & \multirow{2}{*}{ СМ } & \multirow{2}{*}{ СП } & \multirow{2}{*}{$\mathrm{p}$} & \multicolumn{2}{|c|}{ Относительные частоты } & \multirow{2}{\text{U}}{ наблюдений } \\
\cline { 5 - 6 } & & & & $\mathrm{CM}$ & $\mathrm{C \Pi}$ & $-2,8589$ \\
\hline F-10.2 & 3 & 15 & 0,0412 & 0,0138 & 0,0682 & 3,0960 \\
\hline F-21 & 18 & 4 & 0,0503 & 0,0829 & 0,0182 & . \\
\hline
\end{tabular}

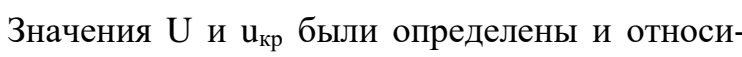
тельно других рубрик МКБ-10. Для рубрики F10.2 $\mathrm{U}=-2,859<\mathrm{u}_{\text {кр}}=-1,645$. Следовательно, гипотеза Н0 не принимается, на этом основании можно считать, что во всей генеральной совокупности вероятность поступления пациентов с СМ в категории F10.2 меньше вероятности поступления пациентов с СП. Для рубрики F21 U=3,0960> $\mathrm{u}_{\mathrm{\kappa p}}=1,645$. Значит гипотеза Н0 не принимается, в результате можно считать, что во всей генеральной совокуп- ности вероятность поступления пациентов с СМ в категории F21 больше вероятности поступления пациентов с СП.

Далее был изучен характер первичных и повторных госпитализаций пациентов с суицидальным поведением.

Для оценки взаимосвязи типа госпитализации пациентов (первичная или повторная) с видом суицидального поведения (СП или СМ) вычислено значение критерия Пирсона $\chi^{2}$ (табл. 4).

T а б л и ц а 4. Расчетные данные для определения зависимости вида госпитализации от типа суицидального поведения пациентов ГБУЗ КО «КОПБ им. А.Е. Лифшица» в 2017-2018 гг.

\begin{tabular}{|c|c|c|c|c|c|}
\hline \multirow{2}{*}{$\begin{array}{c}\text { Кластер } \\
\text { госпитализации }\end{array}$} & \multicolumn{2}{|c|}{ Observed(O) } & \multicolumn{2}{c|}{ Expected (E) } \\
\cline { 2 - 6 } & Первичные & Повторные & Всего & Первичные & Повторные \\
\hline СП & 110 & 108 & 218 & 87,7 & 130,3 \\
\hline СМ & 65 & 152 & 217 & 87,3 & 129,7 \\
\hline Всего & 175 & 260 & 435 & 175 & 260 \\
\hline
\end{tabular}

Ожидаемые значения (Expected) рассмотрены далее в двух направлениях. Рассчитаны ожидае- мые значения для первичных, повторных и общего числа поступлений в кластерах СП и СМ.

\begin{tabular}{|c|c|c|c|}
\hline $\begin{array}{c}\text { Кластер } \\
\text { госпитализации }\end{array}$ & $\begin{array}{c}\text { Ожидаемые значения } \\
\text { первичных поступлений }\end{array}$ & $\begin{array}{c}\text { Ожидаемые значения } \\
\text { повторных поступлений }\end{array}$ & $\begin{array}{c}\text { Ожидаемые значения } \\
\text { всех поступлений }\end{array}$ \\
\hline $\mathrm{CП}$ & $(\mathrm{a}+\mathrm{b})(\mathrm{a}+\mathrm{c}) /(\mathrm{a}+\mathrm{b}+\mathrm{c}+\mathrm{d})$ & $(\mathrm{a}+\mathrm{b})(\mathrm{b}+\mathrm{d}) /(\mathrm{a}+\mathrm{b}+\mathrm{c}+\mathrm{d})$ & $(\mathrm{a}+\mathrm{b})$ \\
\hline $\mathrm{CM}$ & $(\mathrm{a}+\mathrm{c})(\mathrm{c}+\mathrm{d}) /(\mathrm{a}+\mathrm{b}+\mathrm{c}+\mathrm{d})$ & $(\mathrm{b}+\mathrm{d})(\mathrm{c}+\mathrm{d}) /(\mathrm{a}+\mathrm{b}+\mathrm{c}+\mathrm{d})$ & $(\mathrm{c}+\mathrm{d})$ \\
\hline Всего & $(\mathrm{a}+\mathrm{c})$ & $(\mathrm{b}+\mathrm{d})$ & $(\mathrm{a}+\mathrm{b}+\mathrm{c}+\mathrm{d})$ \\
\hline
\end{tabular}


Далее было вычислено наблюдаемое значение

$$
\chi_{\text {набл. }}^{2}=\sum \frac{\left(O_{i j}-E_{i j}\right)^{2}}{E_{i j}}
$$

Соответственно критерий Пирсона $\chi^{2}$ числа наблюдений составляет 19,02. В дальнейшем по таблицам вычислено критическое значение критерия Пирсона $\chi^{2}$, составившее 3,841 , при уровне значимости $\mathrm{p}=0,05$ и числе степеней свободы $\mathrm{f}=(\mathrm{r}-1)(\mathrm{c}-1)=(2-1)(2-1)=1$. При сравнении значения критерия Пирсона $\chi^{2}$ с критическим значением при числе степеней свободы $f$ полученное значение критерия Пирсона $\chi^{2}$ превышает критиче- ское значение критерия Пирсона $\chi^{2}$. На этом основании с вероятностью 0,95 тип госпитализации (первичная или повторная) связан с видом суицидального поведения пациентов. Так, при первичных госпитализациях чаще наблюдались суицидальные попытки и самоповреждения, а при повторных - суицидальные мысли.

При использовании такой встроенной функции Excel, как ХИ2ТЕСТ, используемой для вычисления вероятности конкурирующей гипотезы, ХИ2ТЕСТ $=1,29536 \mathrm{E}-05<0,05$. Подобным образом в последующем рассмотрена каждая рубрика в отдельности (табл. 5).

Т а б л и ц а 5. Распределение госпитализаций пациентов с СП и СМ в ГБУЗ КО «КОПБ им.

А.Е. Лифшица» в 2017-2018 гг. в зависимости от первичных/повторных госпитализаций, кол-во случаев

\begin{tabular}{|c|c|c|c|c|}
\hline \multirow{2}{*}{ Рубрика МКБ-10 } & \multicolumn{2}{|c|}{ Первичные поступления } & \multicolumn{2}{c|}{ Повторные поступления } \\
\cline { 2 - 5 } & СП СМ & 21 & СМ \\
\hline F00-F09 & 20 & 11 & 12 & 11 \\
\hline F10-F19 & 27 & 5 & 53 & 73 \\
\hline F20-F29 & 20 & 17 & 5 & 13 \\
\hline F30-F39 & 11 & 2 & 1 & 1 \\
\hline F40-F48 & 10 & 6 & 0 & 0 \\
\hline F50-F59 & 0 & 1 & 4 & 22 \\
\hline F60-F69 & 6 & 3 & 8 & 0 \\
\hline F70-F79 & 0 & 7 & 0 & 6 \\
\hline F80-F89 & 0 & 0 & 4 & 108 \\
\hline F90-F98 & 16 & 13 & & 152 \\
\hline Bсего & 110 & 65 & & \\
\hline
\end{tabular}

Согласно полученному материалу, пациенты с диагнозом умственной отсталости (F70-F79) формируют большой массив повторных поступлений $(\mathrm{n}=30)$, в группе первичных их меньше $(\mathrm{n}=7)$. Выявлена большая разница в поступлениях пациентов с эмоциональными расстройствами и расстройствами поведения, начинающимися в детском и подростковом возрасте (F90-F98), большую часть составляют первичные госпитализации (29 против 10). Среди пациентов с невротическими, связанными со стрессом и соматоформными расстройствами (F40-F48) преобладали первичные госпитализации (16 против 2). У пациентов с расстройствами настроения (аффективными расстройствами) (F30-F39) первичные гос- питализация чаще встречаются в группе СП (11 СП и 2 СМ), а повторные поступления - чаще в группе с СМ (5 СП и 13 СМ). При психических расстройствах и расстройствах поведения, связанных с употреблением психоактивных веществ (F10-F19), при первичной госпитализации преобладают СП (27 против 5). При шизофрении, шизотипических и бредовых расстройствах (F20F29) отмечается преобладание повторных госпитализаций (126 против 37). В группе органических, включая симптоматические, психические расстройства (F00-F09), при повторных госпитализациях выше доля поступлений с СМ.

Для анализа статистической значимости различий проанализированы данные таблицы 6.

Т а б л и ц а 6. Расчетные данные для определения зависимости вида госпитализации пациентов в ГБУЗ КО «КОПБ им. А.Е. Лифшица» в 2017-2018 гг. от диагностической рубрики МКБ-10

\begin{tabular}{|c|c|c|}
\hline Код по МКБ-10 & $\chi^{2}$ наблюдений & Вероятность конкурирующей гипотезы \\
\hline F00-F09 & 2,647 & 0,104 \\
\hline F10-F19 & 6,727 & 0,009 \\
\hline F20-F29 & 1,663 & 0,197 \\
\hline F30-F39 & 9,764 & 0,002 \\
\hline F40-F48 & 0,117 & 0,732 \\
\hline F50-F59 & - & - \\
\hline F60-F69 & 0,280 & 0,597 \\
\hline F70-F79 & 2,382 & 0,123 \\
\hline F80-F89 & - & - \\
\hline F90-F98 & 0,685 & 0,408 \\
\hline
\end{tabular}


Как продемонстрировано в таблице 6, получены статистически достоверные зависимости лишь по следующим рубрикам: 1) психические расстройства и расстройства поведения, связанные с употреблением психоактивных веществ; 2) расстройства настроения (аффективные расстройства).

Таким образом, в двух рубриках МКБ-10, а именно при расстройствах настроения (аффективных расстройствах) (F30-F39) и психических расстройствах и расстройствах поведения, связанных с употреблением психоактивных веществ (F10-F19), отмечается зависимость частоты первичной или повторной госпитализации от типа суицидального поведения.

\section{ОБСУЖДЕНИЕ}

Согласно литературным данным, самыми распространёнными психиатрическими диагнозами у суицидентов являются личностные, аффективные расстройства и расстройства, связанные со злоупотреблением психоактивных веществ (ПАВ) [17]. При этом наибольший вклад в смертность от суицидов вносят депрессия и другие аффективные расстройства [18]. В случае сочетания депрессии, биполярного аффективного расстройства, посттравматического стрессового расстройства и пограничного расстройства личности с зависимостью от ПАВ суицидальный риск становится еще более очевидным [19]. Отмечена тесная взаимосвязь суицидального поведения с зависимостью от ПАВ, чем обосновано дальнейшее детальное изучение ассоциации суицидального риска с зависимостью от конкретных субстанций [20]. Известно, что в 40-85\% завершенных суицидов им предшествует употребление алкоголя и/или препаратов, воздействующих на ЦНС, даже в случае отсутствия установленного диагноза зависимости от ПАВ [21]. Подчеркивается значимость рациональных профилактических стратегий, направленных на сокращение потребления ПАВ среди населения и в группах риска. При этом стоит отметить, что в странах со сдерживающей алкогольной политикой уровни суицидов среди лиц с зависимостью ниже, но при сохранении более высокого риска [22].

Приводятся данные о том, что более четверти всех госпитализаций в психиатрический стационар с суицидальными попытками приходится на пациентов с шизофренией [23]. От 40\% до 50\% всех пациентов с шизофренией отмечают суицидальные мысли [24], а риск суицида, ассоциированный с данной рубрикой, в 12 раз выше, чем в общей популяции [25]. Высокий процент госпитализаций в рубрике F20-F29 подчеркивает необходимость тщательной оценки суицидального риска, интенсивного лечения и динамичного наблюдения в данной группе пациентов [26].
В случае профилактики суицидов у пациентов с умственной отсталостью к вышеперечисленным мероприятиям уместно добавить образовательные программы для лиц, осуществляющих уход, волонтеров и социальных работников [27]. Наряду с высокой значимостью поддержки со стороны общины [27] обсуждается распространенность коморбидных психических расстройств у лиц с умственной отсталостью, в частности депрессии [28]. Приводятся более высокие показатели суицидальной смертности у лиц с неврологическими расстройствами, в частности боковым амиотрофическим склерозом, болезнью Гентингтона, рассеянным склерозом, последствиями черепномозговых травм, острым нарушением мозгового кровообращения и эпилепсией. Среди возможных профилактических стратегий в группе пациентов с органическими и симптоматическими психическими расстройствами приоритетным направлением признается оптимизация и развитие реабилитационных программ, улучшение лечения и усиление социальной поддержки [29].

Несмотря на небольшой удельный вес иных диагностических рубрик, в исследованной выборке ранее отмечена ассоциированность суицидального риска с личностными расстройствами [30], расстройствами аутистического спектра [31], синдромом дефицита внимания и гиперактивности [32], деменцией [33], обсессивнокомпульсивным расстройством [34], невротическими и аддиктивными расстройствами [35]. Отмечается повышенный риск суицидального поведения при тревожных расстройствах [36] и посттравматическом стрессовом расстройстве, актуальность которого постоянно возрастает за счет терроризма, войн, миграционных процессов и пр. [37].

\section{ЗАКЛЮЧЕНИЕ}

Фактический материал данного исследования дает общее представление о наиболее распространенных психических расстройствах, сопровождающих суицидальное поведение. Высокий удельный вес диагностической рубрики F20-F29 указывает на необходимость развития реабилитационных мероприятий, практики ранней терапевтической интервенции, интенсификации лечебных мероприятий и усиления социальной поддержки пациентов. Детальное изучение суицидального поведения в диагностической рубрике F00-F09 должно сопровождаться оценкой сопутствующей терапии неврологических заболеваний, реабилитации, качества жизни и ухода за пациентами. При изучении суицидального поведения, ассоциированного с диагнозом умственной отсталости, желательно дополнительное исследование частоты суицидальных проявлений в возрастном 
аспекте с акцентом на детско-подростковый контингент.

Обращает на себя внимание большой процент в выборке первичных поступлений пациентов с суицидальным поведением, что подчеркивает актуальность неизбирательных первичных профилактических мероприятий и программ социальной поддержки среди населения. Оптимальными представляются дальнейшие исследования клинико-социальных характеристик суицидентов, а также механизмов суицидального поведения при различных психических расстройствах для улучшения понимания модели и механизмов суицидального поведения популяции региона.

\section{КОНФЛИКТ ИНТЕРЕСОВ}

Авторы заявляют об отсутствии явных и потенциальных конфликтов интересов в связи с публикацией данной статьи.

\section{ИСТОЧНИК ФИНАНСИРОВАНИЯ}

Авторы заявляют об отсутствии спонсорского финансирования при проведении исследования.

\section{СООТВЕТСТВИЕ ПРИНЦИПАМ ЭТИКИ}

Исследование одобрено этическим комитетом при ФГБУ «НМИЦ ПН им. В.П. Сербского» Минздрава России (протокол № 21/2 от 02.04.2018 г.), в рамках выполнения работы по теме кандидатской диссертации «Организация и практика местной суицидологической службы: клиникоэпидемиологический и социально-экономический аспекты (на примере Калужской области) аспиранта ФГБУ «НМИЦ ПН им. В.П. Сербского» Минздрава России Е.С. Носовой.

\section{ЛИТЕРАТУРА/REFERENCES}

1. Boyd A, Van de Velde S, Vilagut G, de Graaf R, O'Neill S, Florescu S, Alonso J, Kovess-Masfety V; EU-WMH Investigators. Gender differences in mental disorders and suicidality in Europe: results from a large cross-sectional population-based study. J Affect Disord. 2015 Mar 1;173:245-54. doi: 10.1016/j.jad.2014.11.002. Epub 2014 Nov 8 . PMID: 25462424.

2. Отдельнова К.А. Определение необходимого числа наблюдений в комплексных социальногигиенических исследованиях. Комплексные социально-гигиенические и клинико-социальные исследования: труды 2-го Московского ГМИ. М., 1980; 6:18-22. Otdelnova K.A. Determination of the required number of observations in complex social and hygienic studies. Complex socio-hygienic and clinical-social research: works of the 2nd Moscow State Medical Institute. Moscow, 1980, 6: 1822 (in Russian).

3. Всемирная организация здравоохранения [Электронный pecypc] (in Russian). World Health Organization [Electronic resource]. URL: https://www.who.int/ru/news-room/factsheets/detail/suicide (дата обращения 11.03.2021)
4. Värnik P. Suicide in the world. Int J Environ Res Public Health. 2012 Mar;9(3):760-71. doi: 10.3390/ijerph9030760. Epub 2012 Mar 2. PMID: 22690161 ; PMCID: PMC3367275.

5. Naghavi M. Global, regional, and national burden of suicide mortality 1990 to 2016: systematic analysis for the Global Burden of Disease Study 2016. BMJ. 2019; 364:194. doi:10.1136/bmj.194

6. Arsenault-Lapierre G, Kim C, Turecki G. Psychiatric diagnoses in 3275 suicides: a meta-analysis. BMC Psychiatry. 2004 Nov 4;4:37. doi: 10.1186/1471244X-4-37. PMID: 15527502; PMCID: PMC534107.

7. Afifi TO, Boman J, Fleisher W, Sareen J. The relationship between child abuse, parental divorce, and lifetime mental disorders and suicidality in a nationally representative adult sample. Child Abuse Negl. 2009 Mar;33(3):139-47. doi: 10.1016/j.chiabu.2008.12.009. Epub 2009 Mar 26. PMID: 19327835.

8. $\mathrm{Xu} \mathrm{Z,} \mathrm{Müller} \mathrm{M,} \mathrm{Heekeren} \mathrm{K}$, Theodoridou A, Metzler S, Dvorsky D, Oexle N, Walitza S, Rössler W, Rüsch N. Pathways between stigma and suicidal ideation among people at risk of psychosis. Schizophr Res. 2016 Apr;172(1-3):184-8. doi: 10.1016/j.schres.2016.01.048. Epub 2016 Feb 1. PMID: 26843510.

9. Stickley A, Koyanagi A. Loneliness, common mental disorders and suicidal behavior: Findings from a general population survey. J Affect Disord. 2016 Jun;197:81-7. doi: 10.1016/j.jad.2016.02.054. Epub 2016 Mar 2. PMID: 26971125.

10. Oexle N, Waldmann T, Staiger T, Xu Z, Rüsch N. Mental illness stigma and suicidality: the role of public and individual stigma. Epidemiol Psychiatr Sci. 2018 Apr;27(2):169-175. doi: 10.1017/S2045796016000949. Epub 2016 Dec 6. PMID: 27919303; PMCID: PMC6998948.

11. Auerbach RP, Mortier P, Bruffaerts R, Alonso J, Benjet C, Cuijpers P, Demyttenaere K, Ebert DD, Green JG, Hasking P, Lee S, Lochner C, McLafferty M, Nock MK, Petukhova MV, Pinder-Amaker S, Rosellini AJ, Sampson NA, Vilagut G, Zaslavsky AM, Kessler RC; WHO WMH-ICS Collaborators. Mental disorder comorbidity and suicidal thoughts and behaviors in the World Health Organization World Mental Health Surveys International College Student initiative. Int J Methods Psychiatr Res. 2019 Jun;28(2):e1752. doi: 10.1002/mpr.1752. Epub 2018 Nov 18. PMID: 30450753; PMCID: PMC6877246.

12. Бохан Н.А., Стоянова И.Я., Счастный Е.Д., Королев А.А. Патопсихологические характеристики пациентов с двойным диагнозом в контексте суицидального поведения. Суицидология. 2014. T. 5, № 2 (15). C. 55-59. Bokhan N.A., Stoyanova I.Ya., Schastnyy E.D., Korolev A.A. Pathopsychological characteristics of patients with a dual diagnosis in the context of suicidal behavior. Suicidology. 2014;5,2(15):55-59 (in Russian). 
13. Kelleher I, Ramsay H, DeVylder J. Psychotic experiences and suicide attempt risk in common mental disorders and borderline personality disorder. Acta Psychiatr Scand. 2017 Mar;135(3):212-218. doi: 10.1111/acps.12693. PMID: 28185269.

14. Всемирная организация здравоохранения [Электронный ресурс] (in Russian). World Health Organization [Electronic resource]. URL: https://covid19.who.int (дата обращения 11.03.21)

15. Sher L. The impact of the COVID-19 pandemic on suicide rates. QJM. 2020 Oct 1;113(10):707-712. doi: 10.1093/qjmed/hcaa202. PMID: 32539153; PMCID: PMC7313777.

16. Gunnell D, Appleby L, Arensman E, Hawton K, John A, Kapur N, Khan M, O'Connor RC, Pirkis J; COVID-19 Suicide Prevention Research Collaboration. Suicide risk and prevention during the COVID-19 pandemic. Lancet Psychiatry. 2020 Jun;7(6):468-471. doi: 10.1016/S22150366(20)30171-1. Epub 2020 Apr 21. PMID: 32330430; PMCID: PMC7173821.

17. Chesney E, Goodwin GM, Fazel S. Risks of allcause and suicide mortality in mental disorders: a meta-review. World Psychiatry. 2014 Jun;13(2):153-60. doi: 10.1002/wps.20128. PMID: 24890068; PMCID: PMC4102288.

18. Hirokawa S, Kawakami N, Matsumoto T, Inagaki A, Eguchi N, Tsuchiya M, Katsumata Y, Akazawa M, Kameyama A, Tachimori H, Takeshima T. Mental disorders and suicide in Japan: a nationwide psychological autopsy case-control study. J Affect Disord. 2012 Oct;140(2):168-75. doi: 10.1016/j.jad.2012.02.001. Epub 2012 Mar 3. PMID: 22391515.

19. Yuodelis-Flores C, Ries RK. Addiction and suicide: A review. Am J Addict. 2015 Mar;24(2):98-104. doi: 10.1111/ajad.12185. Epub 2015 Feb 2. PMID: 25644860.

20. Poorolajal J, Haghtalab T, Farhadi M, Darvishi N. Substance use disorder and risk of suicidal ideation, suicide attempt and suicide death: a meta-analysis. J Public Health (Oxf). 2016 Sep;38(3):e282-e291. doi: 10.1093/pubmed/fdv148. Epub 2015 Oct 26. PMID: 26503486.

21. Vijayakumar L, Kumar MS, Vijayakumar V. Substance use and suicide. Curr Opin Psychiatry. 2011 May;24(3):197-202. doi: 10.1097/YCO.0b013e3283459242. PMID: 21430536.

22. Bachmann S. Epidemiology of Suicide and the Psychiatric Perspective. Int J Environ Res Public Health. 2018 Jul 6;15(7):1425. doi: 10.3390/ijerph15071425. PMID: 29986446 ; PMCID: PMC6068947.

23. Abdeen MS, Shaker NM, Elrassas HH, Hashim MA, Abo Zeid MY. Characteristics of the schizophrenia suicide attempts in comparison with the suicide attempts with other diagnosed psychiatric disorders: An Egyptian study. Int J Soc Psychiatry. 2019 Aug;65(5):368377. doi: 10.1177/0020764019852654. Epub 2019 May 30. PMID: 31144567.
24. Mauri MC, Paletta S, Maffini M, Moliterno D, Altamura AC. Suicide attempts in schizophrenic patients: clinical variables. Asian J Psychiatr. 2013 Oct;6(5):421-7. doi: 10.1016/j.ajp.2013.07.001. Epub 2013 Aug 2. PMID: 24011691.

25. Saha S, Chant D, McGrath J. A systematic review of mortality in schizophrenia: is the differential mortality gap worsening over time? Arch Gen Psychiatry. 2007 Oct;64(10):1123-31. doi: 10.1001/archpsyc.64.10.1123. PMID: 17909124.

26. Togay B, Lippmann S. Suicide Prevention and Schizophrenia. Prim Care Companion CNS Disord. 2019 Sep 26;21(5):19102433. doi: 10.4088/PCC.19102433. PMID: 31577395.

27. Merrick J, Merrick E, Lunsky Y, Kandel I. Suicide Behavior in Persons with Intellectual Disability. The Scientific World Journal. 2005;5:729-735. doi:10.1100/tsw.2005.91

28. Hurley AD, Folstein M, Lam N. Patients with and without intellectual disability seeking outpatient psychiatric services: diagnoses and prescribing pattern. J Intellect Disabil Res. 2003 Jan;47(Pt 1):39-50. doi: 10.1046/j.1365-2788.2003.00463.x. PMID: 12558694.

29. Erlangsen A, Stenager E, Conwell Y, Andersen PK, Hawton K, Benros ME, Nordentoft M, Stenager E. Association Between Neurological Disorders and Death by Suicide in Denmark. JAMA. 2020 Feb 4;323(5):444-454. doi: 10.1001/jama.2019.21834. PMID: 32016308; PMCID: PMC7042859.

30. Paris J. Suicidality in Borderline Personality Disorder. Medicina (Kaunas). 2019 May 28;55(6):223. doi: 10.3390/medicina55060223. PMID: 31142033; PMCID: PMC6632023.

31. Zahid S, Upthegrove R. Suicidality in Autistic Spectrum Disorders: A Systematic Review. Crisis. 2017;38(4):237-246. doi:10.1027/02275910/a000458

32. Stickley A, Koyanagi A, Ruchkin V, Kamio Y. Attention-deficit/hyperactivity disorder symptoms and suicide ideation and attempts: Findings from the Adult Psychiatric Morbidity Survey 2007. J Affect Disord. 2016 Jan 1;189:321-8. doi: 10.1016/j.jad.2015.09.061. Epub 2015 Oct 22. PMID: 26469299.

33. Koyama A, Fujise N, Matsushita M, Ishikawa T, Hashimoto M, Ikeda M. Suicidal ideation and related factors among dementia patients. J Affect Disord. 2015 Jun 1;178:66-70. doi: 10.1016/j.jad.2015.02.019. Epub 2015 Mar 5. PMID: 25795538.

34. Alonso $\mathrm{P}$, Segalàs C, Real E, Pertusa A, Labad J, Jiménez-Murcia S, Jaurrieta N, Bueno B, Vallejo J, Menchón JM. Suicide in patients treated for obsessive-compulsive disorder: a prospective follow-up study. J Affect Disord. 2010 Aug;124(3):300-8. doi: 10.1016/j.jad.2009.12.001. Epub 2010 Jan 13. PMID: 20060171.

35. Бохан Н.А., Воеводин И.В., Мандель А.А. Формирование суицидальности у молодых пациентов с аддиктивными и невротическими рас- 
стройствами: социально-психологические факторы риска и психотерапия. Суицидология. 2020. T. 11, № 2 (39). C. 66-81. Bokhan N.A., Voevodin I.V., Mandel A.A. Formation of suicidality among the young patients with addictive and neurotic disorders: social and psychological risk factors, and psychotherapy. Suicidology. 2020;11,2(39):66-81. (in Russian). DOI: 10.32878/suiciderus.20-11-02(39)-66-81

36. De La Vega D, Giner L, Courtet P. Suicidality in Subjects With Anxiety or Obsessive-Compulsive and Related Disorders: Recent Advances. Curr Psychia- try Rep. 2018 Mar 28;20(4):26. doi: 10.1007/s11920-018-0885-z. PMID: 29594718.

37. Panagioti M, Gooding PA, Tarrier N. A metaanalysis of the association between posttraumatic stress disorder and suicidality: the role of comorbid depression. Compr Psychiatry. 2012 Oct;53(7):91530. doi: 10.1016/j.comppsych.2012.02.009. Epub 2012 Apr 5. PMID: 22483367.

Поступила в редакцию 02.03.2021

Утверждена к печати 28.05.2021

Носова Евгения Сергеевна - заведующий отделением ГБУЗ КО «Калужская областная психиатрическая больница им. А.Е. Лифшица». SPIN-код 3224-1027. ORCID iD 0000-0002-8275-7749. ResearcherID W-38012017.

Жуков Игорь Вячеславович - главный врач ГБУЗ КО «Калужская областная психиатрическая больница им. A.Е. Лифшица». ORCID iD 0000-0001-7081-6636. ResearcherID AAH-7820-2019. Zhukov_kopb@mail.ru

Радулов Семён Пантелеевич - заместитель главного врача ГБУЗ КО «Калужская областная психиатрическая больница им. A.Е. Лифшица». ORCID iD 0000-0003-2022-9171. ResearcherID AAH-7830-2019. seomara@mail.ru

Носова Евгения Сергеевна, nosova.evgenya@lenta.ru 
UDC 616.89-008.1:616.89-008.441.44(470.3)(=1.2)(06)

For citation: Nosova E.S., Zhukov I.V., Radulov S.P. Mental disorders and suicidal behavior: the results of the analysis of medical records. Siberian Herald of Psychiatry and Addiction Psychiatry. 2021; 2 (111): 54-63. https://doi.org/10.26617/1810-3111-2021-2(111)-54-63

\title{
Mental disorders and suicidal behavior: the results of the analysis of medical records
}

\section{Nosova E.S., Zhukov I.V., Radulov S.P.}

Kaluga Regional Psychiatric Hospital named after A.E. Lifshitz

Mayakovsky Street 55, 248009, Kaluga, Russian Federation

\begin{abstract}
Mental disorders are one of the most studied risk factors for suicidal behavior. The severity of suicidal risk in mental illness varies depending on the diagnosis and demographic characteristics [1], which indicates a direction for further research on the issue at the local level. Objective: to study the nosological structure of admissions to a psychiatric hospital in connection with suicidal behavior and to identify vulnerable groups for the development of preventive measures. Materials and Methods: based on the analysis of archival medical documentation of the Kaluga Regional Psychiatric Hospital named after A.E. Lifshitz for 2017-2018, 435 cases of hospitalizations of patients with suicidal behavior were studied. When forming the sample, the technique of K.A. Otdelnova (1980) was used for the study of increased accuracy $(\mathrm{t}=2.0 ; \mathrm{p}=0.95)$ [2]. Statistical data processing was carried out using statistical tables and Microsoft Excel. Results: in terms of frequency of occurrence, diagnostic rubrics were arranged as follows: F20-F29 (37.47\%), F00-F09 (17.7\%), F10-F19 (12.64\%), F90-F98 (8.97\%), F70-F79 (8.5\%), F30-F39 (7.13\%), less than 10\% - other headings. It has been proven that rubric F70-F79 and rubric F21 were statistically significantly associated with the risk of hospitalization with suicidal thoughts, code F10.2 - with the risk of a suicidal attempt. Initial admissions were more often observed in the group of patients with suicidal attempts, repeated - in the group of patients with suicidal thoughts. However, from the point of view of the diagnosis, the dependence was statistically significant only in the rubrics F30-F39 and F10-F19; the primary admissions of patients with these psychiatric diagnoses were more often due to suicidal attempts. Conclusions. The discussed research evidence provides an overview of the most common mental disorders associated with suicidal behavior in hospitalized patients. The presented nosological structure of hospitalizations determines the need to develop rehabilitation measures, optimize therapeutic interventions and strengthen social support for patients. Further studies of the clinical and social characteristics of suicides, as well as the mechanisms of suicidal behavior in various mental disorders, are optimal to improve understanding of the model of suicidal behavior of the population of the region using the example of the population of Kaluga, which is part of the Central Federal District.

Keywords: mental and behavioral disorders, suicide, suicidal thoughts, suicidal attempt, epidemiology, suicidal
\end{abstract} behavior, risk factors.

Received March 02.2021

Accepted May 28.2021

Nosova Evgenia S. - Head of the Unit, State Budgetary Healthcare Institution of the Kaluga Region "Kaluga Regional Psychiatric Hospital named after A.E. Lifshitz". SPIN-code 3224-1027. ORCID iD 0000-0002-8275-7749. ResearcherID W-3801-2017.

Zhukov Igor V. - chief physician, State Budgetary Healthcare Institution of the Kaluga Region "Kaluga Regional Psychiatric Hospital named after A.E. Lifshitz". ORCID iD 0000-0001-7081-6636. ResearcherID AAH-7820-2019. Zhukov_kopb@mail.ru

Radulov Semen P. - Deputy Chief Physician, State Budgetary Healthcare Institution of the Kaluga Region "Kaluga Regional Psychiatric Hospital named after A.E. Lifshitz". ORCID iD 0000-0003-2022-9171. ResearcherID AAH7830-2019. seomara@mail.ru

Nosova Evgenia S., nosova.evgenya@lenta.ru 